Z. klin. Chem. u. klin. Biochem.

9. Jg., S. $405-410$, September 1971

\title{
Zur quantitativen Bestimmung von Serumproteinen nach Auftrennung mit der Disk-Elektrophorese im Polyacrylamidgel
}

\author{
Von L. Strauch ${ }^{1}$ ) und K.-D. TYMPNER ${ }^{2}$ ) \\ Max-Planck-Institut für Eivveiß- und Lederforscbung, München, und Universitäts-Kinderklinik, München
}

(Eingegangen am 24. März 1971)

\begin{abstract}
Die Autoren beschreiben eine disk-elektrophoretische Methode zur quantitativen Bestimmung von Serumproteinen. Die Elektrophorese erfolgt in $14 \mathrm{~cm}$ langen zylindrischen Gelen unter Anwendung eines diskontinuierlichen alkalischen Ein-Gel-Puffersystems. Nach der Auftrennung werden die Proteinfraktionen mit Amidoschwarz $10 \mathrm{~B}$ angefärbt, der überschüssige Farbstoff ausgewaschen und die Trenngele nach dem Transparenzverfahren densitometrisch ausgewertet. Messungen der Gipfelhöhen und der Flächenintegrale ergeben bei Albumin und IgG, die als Testsubstanzen herangezogen wurden, in dem zur Auswertung gelangendem Mengenbereich einen linearen Verlauf der Konzentrationsabhängigkeit. Anhand der Ergebnisse erscheint es möglich, die Disk-Elektrophorese nicht nur als qualitative, sondern auch als eine quantitative Analysenmethode einzusetzen.
\end{abstract}

\section{Quantitative determination of serum proteins following their separation by disc-electrophoresis in polyacrylamide gel}

A disc-electrophoretic method is described for the quantitative determination of serum proteins. The electrophoresis is performed in cylindrical gels up to $14 \mathrm{~cm}$ long, using a discontinuous alkaline buffer system. After the separation, the proteins are stained with amidoblack $10 \mathrm{~B}$, the excess dye is removed by washing, and the running gel evaluated by the measurement of transparency in a densito meter. Peak heights and area integrals show a linear relationship with concentration in the measureable range when IgG-globulin and albumin are used as test substances. The results show that disc-electrophoresis may be used for both qualitative and quantitive analysis.

Die außerordentlich scharfe Trennung, die mit der Disk-Elektrophorese im Polyacrylamid erreicht werden kann, erlaubt uns bei der Analyse von Proteingemischen einen viel tieferen Einblick in die Verteilung der einzelnen Proteinfraktionen, als dies mit Methoden der Papier-, Celluloseacetatfolien- oder Agar-Elektrophorese bisher möglich war (1).

Auftrennungen von Proteinen aus biologischen Flüssigkeiten mit Hilfe der Disk-Elektrophorese haben in der klinischen Forschung bereits eine Bedeutung erlangt. Die Methode wurde zur Beurteilung von Krankheitsverläufen mit Erfolg herangezogen, wie Untersuchungen bei akuten und chronischen Erkrankungen der Leber (2) und bei Patienten mit einem Malignom (3) gezeigt haben.

Disk-elektrophoretische Auftrennungen von Serumproteinen ergeben in Kombination mit anderen klinisch-chemischen Untersuchungsergebnissen Hinweise, die prophylaktische Konisequenzen bei Herz- und Gefäßerkrankungen nach sich ziehen (4). In einigen Fällen lassen sich mit dieser Methode auch genetische Besonderheiten oder pathologische Veränderungen in der Verteilung von Serumproteinen besonders leicht erkennen $(1,5)$.

Analog zur Papierelektrophorese unterscheidet man verschiedene disk-elektrophoretisch signifikante Serumtypen $(6,7)$.

Für den Routinebetrieb geeignete disk-elektrophoretische Methoden zur qualitativen Erfassung von

1) Neue Adresse: F. Hoffmann-La Roche \& Co. AG, Abt. VI/ Med., CH-4002 Basel (Schweiz).

2) Mit Unterstützung der Deutschen Forschungsgemeinschaft, Bad Godesberg (TY 1/1).
Lipoproteinämien, zur Typisierung von Hämoglobinen, zur Bestimmung von Isozymen der Lactatdehydrogenase bei Herzinfarkt und Lebererkrankungen, zur Diagnose von Paraproteinämien und humoralen Immundefizienzen, sind in Vorbereitung ${ }^{3}$ ).

Das Bestreben, von einer rein qualitativen Abschätzung zu quantitativen Aussagen zu gelangen, ist auch bei der Disk-Elektrophorese deutlich zu erkennen. Während bei klassischen Elektrophoreseverfahren (8) diese Entwicklung bereits abgeschlossen ist, müssen quantitative Meß- und Auswertungsmethoden in der DiskElektrophorese erst erarbeitet werden. Diese Entwicklung erscheint jedoch weniger beschwerlich, da man einerseits auf zahlreiche Erfahrungen bei den heute schon als "klassisch" zu bezeichnenden Auswertungsverfahren bei der Trägerelektrophorese $(9,10,11,15)$ und bei der Papierchromatographie $(12,13,14)$ zurückgreifen kann und andererseits ein großes Angebot an Densitometern und weiteren Auswertungsgeräten zur Verfügung steht.

Wir untersuchten daher, mit welcher Genauigkeit disk-elektrophoretisch aufgetrennte Serumproteinfraktionen erfaßbar sind. Um sich den Möglichkeiten eines routinemäßigen Betriebs anzunähern, wurde die DiskElektrophorese in zylindrischen Gelen ausgeführt. Die Gele wurden nach der Anfärbung in runder Form in das hier angegebene Densitometergerät eingesetzt; die Intensität der Proteinfraktionen wurde nach dem Transparenz-Verfahren $(1,9)$ gemessen.

Es hat uns vor allem interessiert, bis zu welchen Konzentrationen noch lineare Verhältnisse der Licht-

3) Weitere Mittcilungen: Dr. L. Strauch. 
absorption zu erreichen sind. Bei der Auswertung haben wir uns auf die Serumalbuminbande und auf die Bande der IgG-Globuline beschränkt.

\section{Material und Methoden}

\section{Serumpräparate}

Zur elektrophoretischen Auftrennung wurde ein standardisiertes Humanserum Op. Nr. $166(1,0 \mathrm{ml}=30 \mathrm{mg}$ Albumin) und ein $\gamma$-Globulin-Präparat (1) - Op. Nr. $601(1,0 \mathrm{ml}=160 \mathrm{mg} \gamma$ Globulin) der Behringwrerke AG, Marburg verwendet.

\section{Disk-Elektrophorese}

Apparatur

Die Elektrophorese wurde zuerst in der Trennkammer für die präparative und analytische Disk-Elektrophorese, Modell DEP 100 der Apparatur „Discophor ${ }^{\ll 4}$ ) durchgeführt. Für Routineuntersuchungen wurde weiter die analytische Trennkammer Modell DEA 90 verwendet. Um reproduzierbare Ergebnisse zu bekommen, führten wir die notwendigen Arbeitsschritte mit entsprechenden Geräten und der Ausrüstung für die analytische Disk-Elektrophorese der Modellserie 90 des oben angegebenen Herstellers aus.

\section{Gel- und Puffersystem}

Wir führten die Trennungen im 7,5proz. ( $\mathrm{g} / \mathrm{v}$ ) Polyacrylamidgel durch und verwendeten das diskontinuierliche Ein-Gel-Puffersystem nach Schaub und Strauch (16), welches wir hier in zusammengefaßter Form angeben:

Elektrodenpuffer: $3,0 \mathrm{~g}$ Tris $+14,4 \mathrm{~g}$ Glycin mit $1 \mathrm{M} \mathrm{NaOH}$ gepuffert auf $\mathrm{pH} 9,0$ und mit dest. Wasser auf 11 aufgefüllt.

Gelpuffer: $40,0 \mathrm{~g}$ Tris mit $1 \mathrm{M} \mathrm{HCl}$ auf 8,0 gebracht und mit dest. Wasser auf 11 aufgefüllt.

Stammlösungen: (bei $4^{\circ}$ etwa eine Woche haltbar)

1. $30,0 \mathrm{~g}$ Acrylamid und $0,8 \mathrm{~g} \mathrm{~N}, \mathrm{~N}^{\prime}$-Methylenbisacrylamid gelöst in $100 \mathrm{ml}$ Gelpuffer.

2. $1,6 \mathrm{ml} \mathrm{3-Dimethylaminoproprionitril} \mathrm{gelöst} \mathrm{in} 100 \mathrm{ml}$ Gelpuffer.

3. $0,04 \mathrm{~g}$ Kaliumhexacyanoferrat-[II] gelöst in $100 \mathrm{ml}$ Gelpuffer.

4. 0,48 g Ammoniumperoxodisulfat in $100 \mathrm{ml}$ Gelpuffer.

Trenngellösung: Die Stammlösungen werden in der Reihenfolge $1,2,3,4 \mathrm{zu}$ gleichen Teilen gemischt, mit Hilfe der Wasserstrahlpumpe in einem dickwandigen Gefä $B$ etwa 20 Min. entlüftet, in die Gel-Röhrchen eingefüllt und zur Ausbildung einer geraden Oberfläche beim Polymerisieren mit entlüftetem dest. Wasser oder Gelpuffer überschichtet.

\section{Ausfïbrung der Disk-Elektrophorese ${ }^{5}$ )}

Die sauber gereinigten und getrockneten Elektrophorese-Röhrchen $(14 \mathrm{~cm}$ lang, Innendurchmesser $6 \mathrm{~mm}$ ) wurden mit der Strichmarkierung nach oben in den Polymerisierständer DSP 90 eingesteckt, bis zur Marke (10 cm hoch) mit der Trenngellösung gefüllt und anschließend etwa $2 \mathrm{~cm}$ hoch mit dest. Wasser überschichtet. Zur schnelleren Polymerisierung wurden die GélRöhrchen mit der UV-Lampe DUL 90 bestrahlt. Nach der Polymerisierung wird die Überschichtungsflüssigkeit vorsichtig abgekippt und die Gel-Oberfläche mit der Gel-Pufferlösung zweimal gespült.

4) Hersteller: Wissenschaftlich-Technische Werkstätten, GmbH., 8120 Weilheim/Obb., Trifthofstr. 17; „Discophor“, Disk-Elektrophorese-Automat, Modell EA 100 und Analytische Disk-Elektrophorese-Apparatur Modell EA 90 mit entsprechenden Zusatz-
geräten.

5) Vergleiche weiter: Beschreibung und Bedienungsanleitung für die analytische Disk-Elektrophorese.
Das untere Elektrodengefä $\beta$ der Trennkammer wird mit der Elektrodenpufferlösung gefüllt. Die Elektrophorese-Röhrchen wurden von unten in den Boden des analytischen Aufsatzes der Trennkammer DEP 100 oder der Trennkammer DEA 90 eingesetzt, so daß sie über den Boden etwa $2 \mathrm{~cm}$ herausragten. Die Kühlung wurde in Betrieb gesetzt und der analytische Aufsatz auf das untere Elektrodengefäß aufgesetzt. Die Röhrchen tauchen nun in die Pufferlösung ein. Die Temperatur in der Trennkammer betrug während des Versuches etwa $15-17^{\circ}$.

Die Serumprobe wurde zur Erhöhung der Dichte zu 10\% (g/v) mit Glucose versetzt und mit einer Hamilton-Spritze auf die Geloberfläche aufgetragen. Um die anschließende Anfärbung der Proteine nicht zu stören, wurde kein Bromphenolblau als Gradientmarker verwendet, da der Puffergradient in unserem System mit freiem Auge gut sichtbar ist.

Die Elektrophorese wurde mit $4 \mathrm{~mA} /$ Röhrchen begonnen. Nach der Einwanderung der Probe in das Trenngel erhöhten wir die Stromstärke auf $5 \mathrm{~mA} /$ Röhrchen. Die Spannung war am Anfang etwa $80 \mathrm{~V}$ und erhöhte sich während des Versuches auf etwa $140 \mathrm{~V}$. Nachdem sich der Puffergradient bis auf etwa $3 \mathrm{~mm}$ an das untere Gelende angenähert hatte - das dauerte ungefähr 4 Stdn. - , wurde die Elektrophorese beendet.

Nach der Elektrophorese wurde die Gele sofort auf übliche Weise aus den Röhrchen entfernt und mit Hilfe der Färbevorvorrichtung DFV $9030 \mathrm{Min}$. lang mit 0,5proz. (g/v) Amidoschwarz $10 \mathrm{~B}$ in 7 proz. $(\nabla / \nabla)$ Essigsäure angefärbt. Die Entfärbung erfolgte über Nacht im Universal-Entfärbebad DEB 90 in 1proz. $(\nabla / v)$ Essigsäure unter mechanischer Umwälzung und Regenerierung der Waschflüssigkeit.

\section{Densitometrische Auswertung ${ }^{6}$ )}

Die entfärbten Gele wurden unter Zugabe von 1proz. ( $/ / \mathrm{v})$ Essigsäure blasenfrei in optisch einwandfreie, unten zugeschmolzene Glastöhrchen ( $17 \mathrm{~cm}$ lang, Innendurchmesser $8 \mathrm{~mm}$ ) eingelegt, oben ebenso blasenfrei verschlossen und in das Densitometergerät "Chromoscan" eingesetzt. Die Auswertung erfolgte mit dem Spalt $0,5 \mathrm{~mm}$ bei $620 \mathrm{~nm}$ unter Vorschaltung von Filtern mit einer Gesamtextraktion von 2,5, um die Albuminbande noch voll zu erfassen. Die Basislinie wurde bei jeder Messung auf $2,0 \mathrm{~cm}$ der Diagrammhöhe eingestellt.

Zur Aufzeichnung der Extinktion bzw. der Gipfelhöhe wurde der mit dem Gerät mitgelieferte flachste Kalibrierungskeil mit der Steigung von $50^{\circ}$, verwendet. Auf eine Korrektur und Anpassung der Keile zwecks Erweiterung des Linearitätsbereichs, haben wir absichtlich verzichtet. Die UUbersetzung der Diagrammlänge zur Gellänge betrug 1:1 oder 1:3.

Zur Auswertung wurde im Disk-Elektropherogramm die Basislinie gezogen und in einzelnen Fraktionen die entsprechenden Gauss-Kurven eingezeichnet. Von der Gipfelspitze wurde auf die Basislinie eine senkrechte Gerade, die bei asymmetrischen Proteinverteilungen nicht in der Mitte der Gauss-Kurve zu verlaufen braucht, gezogen und die Gipfelhöhe ausgemessen. Die Bestimmung der Gipfelfläche wurde auf übliche Weise mit einem Planimetergerät. (Fa. A. Ott, Kempten, BRD) vorgenommen. Die Integrieranordnung des Densitometergerätes wurde bei der Auswertung von Disk-Elektrophoresen nicht verwendet.

\section{Ergebnisse}

Zur Ermittlung der Eichkurve für Serumalbumin wurde standardisiertes Humanserum verwendet. Eine Versuchsreihe, in der steigende Mengen von $1-10 \mu l$ Serum aufgetragen und disk-elektrophoretisch aufgetrennt wurden, ist in Abbildung 1 wiedergegeben.

6) "Chromoscan", Double beam recording and integrating Densitometer; Hersteller: Joyce-Loebl \& Co., Ltd., Gateshead-onTyne, 11, England, Vertretung für die Bundesrepublik Deutschland, Joyce-Loebl Gerätetechnik GmbH, 7769 Kalkhofen/Bodensee, Romhalden. 
Eine Auswertung der Albuminbanden ergab die in Abbildung 2 dargestellten Eichkurven.

Die in Abbildung 3 angegebene Eichkurve für IgG wurde aus einer analog ausgeführten Versuchsreihe, bei der das $\boldsymbol{\gamma}$-Globulin-Präparat disk-elektrophoretisch aufgetrennt wurde, gewonnen.

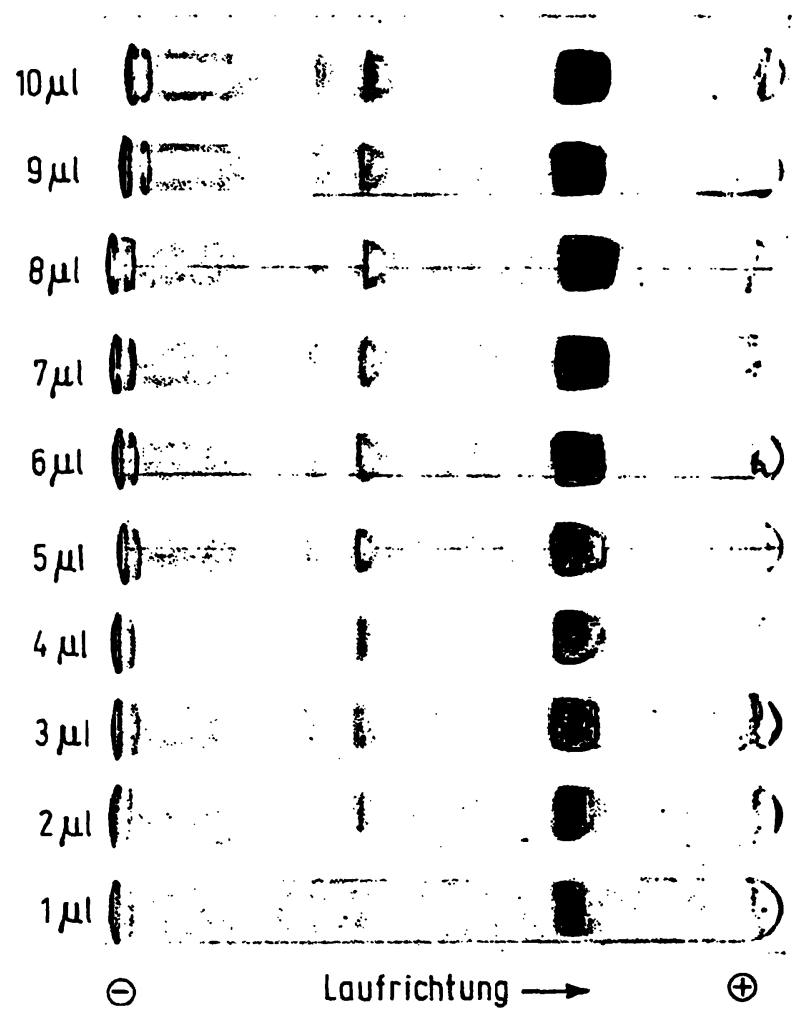

Abb. 1

Disk-elektrophoretische Auftrennung steigender Mengen von standardisiertem Humanserum. Gel-Länge $10 \mathrm{~cm}$
Als Beispiel für die quantitative Bestimmung wird hier die disk-elektrophoretische Analyse einer Serumprobe gezeigt.

Die Proteinverteilung ist in Abbildung 4 angegeben. In der Tabelle 1 sind die Ergebnisse der beiden Auswertungsmethoden gegenübergestellt.

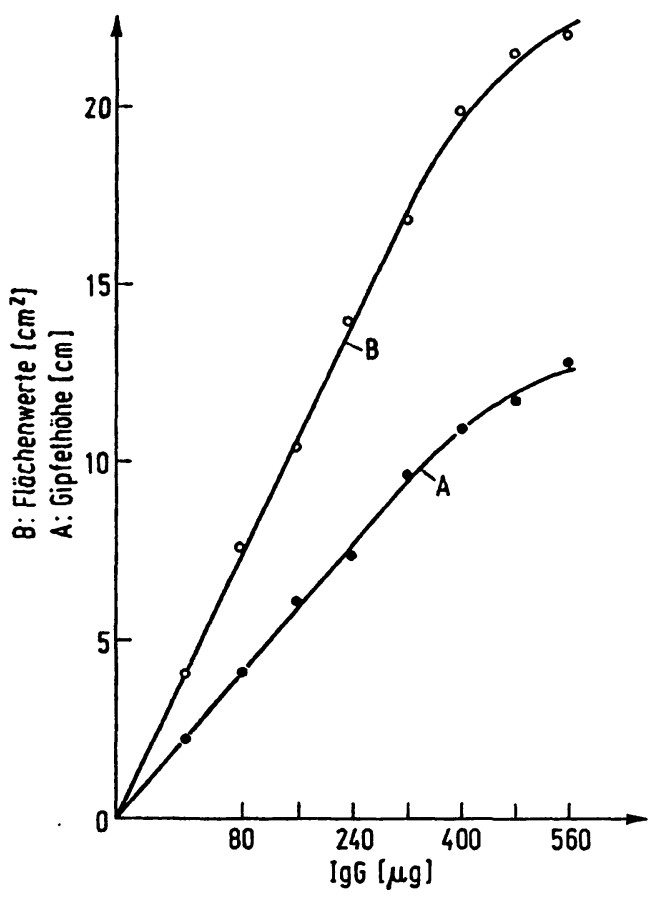

Abb. 3

Eichkurve für die Auswertung steigender Mengen IgG-Globulin. Kurve A - Methode der maximalen Gipfelhöhe; Kurve B - Methode der Flächenintegrale (Mittelwerte aus 5 Bestimmungen)

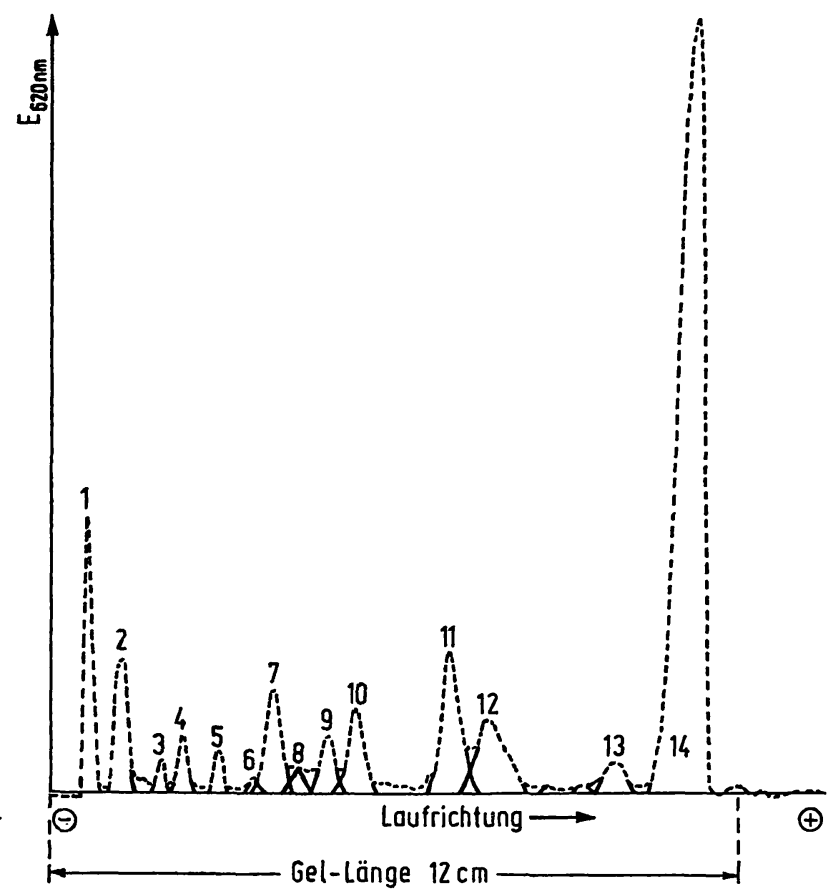

Abb. 4

Disk-Elektropherogramm eines Humanserums Pat.: SCH. M., geb. 11. 12. 1960, Diagnose: Agammaglobulinämie)

Eichkurven für die Auswertung steigender Mengen Albumin. Densitometerkurven der Albuminbanden aus der Abb. 1 . Kurve A-Methode der maximalen Gipfelhöhe; Kurve $\mathrm{B}-$ Methode
integrale (Mittelwerte aus 5 Bestimmungen) 
Tab. 1

Die relativen Anteile einzelner Serumproteinfraktionen nach Auftrennung mit der Disk-Elektrophorese. Serumprobe aus der Abbildung 4 (Mittelwerte aus 5 Bestimmungen)

\begin{tabular}{|c|c|c|c|c|c|}
\hline $\begin{array}{c}\text { Fraktionen } \\
1\end{array}$ & $\begin{array}{l}\text { A-Gip } \\
\text { Höhe mm } \\
\quad 2\end{array}$ & $\begin{array}{l}\text { öhe } \\
\text { rel. \% } \\
3\end{array}$ & $\begin{array}{l}\text { B-Gipfelf } \\
\text { Planimeterwert } \\
4\end{array}$ & $\begin{array}{l}\text { rel. } \% \\
5\end{array}$ & $\begin{array}{c}\text { Zuordnung der einzelnen } \\
\text { Banden }(1,18) \\
6\end{array}$ \\
\hline $\begin{array}{l}1 \\
2\end{array}$ & $\begin{array}{l}49 \\
24\end{array}$ & $\begin{array}{l}15,1 \\
7,3\end{array}$ & $\begin{array}{l}7 \\
6\end{array}$ & $\begin{array}{l}5,8 \\
4,9\end{array}$ & $\begin{array}{l}1-2 \text { (Doppelbanden) } \\
\text { Makroglobuline }\end{array}$ \\
\hline $\begin{array}{l}3 \\
4 \\
5\end{array}$ & $\begin{array}{r}6 \\
10 \\
8\end{array}$ & $\begin{array}{l}1,8 \\
3,0 \\
2,4\end{array}$ & $\begin{array}{l}1 \\
3 \\
1\end{array}$ & $\begin{array}{l}0,8 \\
2,5 \\
0,8\end{array}$ & 3-5 Ḣaptoglobine \\
\hline $\begin{array}{l}6 \\
7 \\
8\end{array}$ & $\begin{array}{r}3 \\
18 \\
4\end{array}$ & $\begin{array}{l}0,9 \\
5,5 \\
1,2\end{array}$ & $\begin{array}{l}1 \\
6 \\
1\end{array}$ & $\begin{array}{l}0,8 \\
4,9 \\
0,8\end{array}$ & $6-8$ Globuline \\
\hline $\begin{array}{r}9 \\
10\end{array}$ & $\begin{array}{l}10 \\
15\end{array}$ & $\begin{array}{l}3,0 \\
4,6\end{array}$ & $\begin{array}{l}3 \\
5,5\end{array}$ & $\begin{array}{l}2,5 \\
4,5\end{array}$ & 9-10 Posttransferrine \\
\hline 11 & 25 & 7,6 & 9 & 7,4 & 11 Transferrin \\
\hline $\begin{array}{l}12 \\
13\end{array}$ & $\begin{array}{r}13 \\
5\end{array}$ & $\begin{array}{l}4,0 \\
1,5\end{array}$ & $\begin{array}{l}8 \\
3,5\end{array}$ & $\begin{array}{l}6,6 \\
2,9\end{array}$ & 12-13 Postalbumine \\
\hline 14 & 138 & 42,2 & 67,0 & 55,0 & 14 Albumin \\
\hline Zusammen & 328 & 100,1 & 122,0 & 100,2 & \\
\hline
\end{tabular}

\section{Diskussion}

Um disk-elektrophoretisch aufgetrennte Proteinbanden mit Hilfe üblicher Densitometergeräte, die eine Auflösung bis zu 0,2 $\mathrm{mm}$ aufweisen, quantitativ auswerten zu können, ist es erforderlich, die Fraktionen weit genug auseinander wandern zu lassen. Das kann man erreichen, in dem man den Wanderungsweg im Rahmen der gegebenen Versuchsanordnung so weit wie möglich zu verlängern versucht. Einer beliebigen Verlängerung des Wanderungsweges wirken hauptsächlich zwei Faktoren entgegen:

1. die Aufhebung des für die Schärfung der Fraktionen verantwortlichen Konzentrierungseffektes, nachdem im Trenngel die Folge-Ionen die Proteine überwandert haben und

2. die nachfolgend eintretende freie Diffusion der Proteinmoleküle, die während der Wanderung zu einer zunehmenden Verbreiterung und evtl. zu einer Überdeckung der benachbarten Fraktionen führen kann.

Das von uns eingeführte Puffersystem (16), mit dem $\mathrm{pH}$ von 9,0 im Elektrodenpuffer und $\mathrm{pH} 8,0 \mathrm{im}$ Gelpuffer, hat zur Folge, daß die Glycinat-Ionen (FolgeIonen) beim Übergang aus der Elektrodenlösung in das Trenngel verzögert werden, die Proteine daher viel später überwandern und dadurch der Konzentrierungseffekt länger aufrechterhalten bleibt. Gleichzeitig wird der Weg, in dem die Proteine im Gebiet einheitlicher Feldstärke und $\mathrm{pH}$-Werte wandern, also einer freien Zonen-Elektrophorese unterliegen, kürzer. Die freie Diffusion der Proteinteilchen wird dadurch unterdrückt, was ebenfalls zu einer größeren Schärfe der Fraktionen beiträgt.

Durch diese Maßnahme war es möglich, den Wanderungsweg auf über $10 \mathrm{~cm}$ zu verlängern und noch scharfe, gut auseinander getrennte Proteinbanden zu erhalten.

Ein grobporiges Sammelgel, wie es von Davis und OrNSTEIN (1) eingeführt wurde, ist bei unserem Puffersystem nicht notwendig. Der Arbeitsaufwand und der Chemikalienbedarf wird dadurch wesentlich verringert.
Die etwas langsamere Wanderungsgeschwindigkeit führt bei unserem Puffersystem, gegenüber dem oben angegebenen, zu einer längeren Laufzeit der Elektrophorese. Da man aber dadurch die Möglichkeit der quantitativen Auswertung mittels relativ preiswerter Densitometergeräte gewinnt, kann das nicht als Nachteil empfunden werden.

Um reproduzierbare Analysenergebnisse zu erhalten, wobei für die Beurteilung der Methodik ein zuverlässig zu reproduzierender "Hauswert" (18) von besonderer Wichtigkeit ist, muß man für eine Vereinheitlichung der einzelnen Analysenschritte sorgen. Eine totale Automatisierung der Disk-Elektrophorese, deren Voraussetzung eine Normung aller für die Analyse notwendigen Arbeitsgänge wäre, wird in absehbarer Zeit kaum zu erreichen sein.

Man kann sich dem erstrebten Zustand annähern und die Analyse weitgehend standardisieren, indem man jeden einzelnen Arbeitsgang mit Hilfe eines speziell für diesen Zweck entwickelten Gerätes auszuführen versucht. Die von uns benutzte Geräteausrüstung erfüllt diese Anforderungen in einer befriedigenden Weise. Die Elektrophorese wird in standardisierten GelRöhrchen aus Präzisionsglas ausgeführt. Alle Arbeitsgänge, wie die Polymerisierung, die Trennung selbst, die Anfärbung und die Entfärbung der Gele können mit den entsprechenden Vorrichtungen unter standardisierten und reproduzierbaren Bedingungen ablaufen. Auch die Auswertung der Bandenintensitäten kann in zufriedenstellender Weise standardisiert werden.

Die Auswertung erfolgte nicht in flachen, sondern in zylindrischen Gelen, also unter relativ ungünstigen optischen Bedingungen. Durch die Linsenwirkung der Probenröhre wurden die Lichtstrahlen auf die Photozelle des Densitometers nämlich nicht parallel, sondern in gebündelter Form geworfen.

Dennoch konnten wir eine lineare Konzentrationsabhängigkeit der Lichtabsorption bis zụ relativ hohen Proteinmengen erreichen. Die Eichkurvé für Albumin (Abb. 2) zeigt nach der Methode der maximalen Gipfel- 
höhe (maximum color density-Methode (19)) einen linearen Verlauf bis zu $75 \mu \mathrm{g}$ (Kurve A). Eine noch höhere Linearität wurde bei der Methode der Flächenintegrale der einzelnen Gipfel erreicht (total color density-Methode (20)), bei der die Eichkurve bis zu über $150 \mu \mathrm{g}$ linear verläuft.

Es ist sehr bemerkenswert, daß bereits 1954 von Grassmann und Hannig (9) bei der Auswertung von Papierelektrophoresen nach dem Transparenzverfahren mit der Methode der Flächenintegrale Eichkurven für steigende Mengen von Serumalbumin beschrieben wurden, die einen linearen Verlauf bis zu $160 \mu \mathrm{g}$ aufweisen (21).

Wir können daher feststellen, daß die von uns beschriebene Methodik der Auswertung in der DiskElektrophorese mit derjenigen bei der Papierelektrophorese vergleichbar ist. Wenn man bei der Aufzeichnung der Proteinbanden korrigierte Kalibrierungskeile verwenden würde, könnte man eine noch höhere Linearität der Konzentrationsabhängigkeit erreichen (22).

Das IgG erscheint bei der Disk-Elektrophorese nicht als eine scharfe Bande, sondern als eine im Bereich der Haptoglobine und $\alpha_{2}$-Globuline liegende diffuse Wolke (23) (Proteinfamilie). Wegen einer solchen Verteilung ist der Geltungsbereich des LAMBERT-BEER'schen-Gesetzes höher als beim Albumin und die Eichkurven zeigen einen linearen Verlauf bis zu etwa $300 \mu \mathrm{g}$ (Kurven A und B, Abb. 3).

Bei höheren Mengen tritt sehr bald eine Krümmung der Eichkurven ein, die bei noch höheren Konzentrationen einen sogar abfallenden Verlauf zeigen (in Abb. 3 nicht eingezeichnet).

Um die beiden Auswertungsmethoden miteinander besser vergleichen zu können, haben wir uns ein Serum, welches keine IgG-Globuline enthalten hat, ausgesucht. So konnten wir ohne störenden Hintergrund auch die Proteinbanden im Bereich der Makroglobuline, Haptoglobine und Globuline (Abb. 4, Banden 1-8) auswerten, da die Minima zwischen den Gipfeln fast auf die Basislinie zurückfallen. Unsere weiteren Überlegungen beruhen auf der bereits von anderen Autoren $(8,9)$ bestätigten Annahme, daß der zur Proteinanfärbung verwendete Farbstoff Amidoschwarz $10 \mathrm{~B}$ von Albumin und Globulin zu gleichen Anteilen gebunden wird. Die. Banden 1-2 sind etwa 0,2 mm dicke Doppelbanden, die vom Densitometer nicht mehr aufgelöst werden können. Die sehr hohe Farbstoffdichte in diesen Banden hat schon im niedrigeren Konzentrationsbereich eine Abweichung von der Linearität zur Folge.

Aus der Tabelle 1 kann man entnehmen, daß beide Methoden durchaus brauchbare Werte liefern, vorausgesetzt die Probenmenge liegt im linearen Eichkurvenbereich. Bei Humanserum entspricht das einem aufgetragenen Probenvolumen von etwa $3-6 \mu$ l Serum pro Röhrchen.

Die Auswertung nach der Methode $\mathrm{A}$ ist schneller als nach der Methode B, die eine Integrierung der Gipfelflächen erfordert. Mit dem von uns verwendeten Densitometergerät war es wegen der hohen Anzahl von Fraktionen, technisch nicht ohne weiteres möglich, die Integrierungsanordnung direkt $\mathrm{zu}$ verwenden. Einer automatischen Integrierung sowie einer ComputerSpeicherung der gewonnenen Werte dürfte bei Benutzung entsprechender Geräte nichts im Wege stehen.

Ein Vergleich der Werte unseres Trennergebnisses mit Ergebnissen anderer Arbeitsgruppen $(17,24)$ ist wegen methodischer Unterschiede und besonders wegen der Tatsache, daß wir aus oben dargestellten Gründen ein pathologisch verändertes Serum angegeben haben, nicht möglich.

Uber die Fehlerbreite der hier angegebenen Methodik können wir zur Zeit, da noch zu wenig statistisch gesicherte Ergebnisse vorliegen, ebenso keine Aussagen machen. Die Fehlerbreite der Methode dürfte jedoch in derselben Größenordnung liegen, wie sie bei ähnlichen Analysenmethoden erreicht werden kann.

Der allgemeinen Verwendbarkeit der Disk-Elektrophorese für die klinische Routine-Diagnostik stehen zur Zeit noch Schwierigkeiten entgegen, da man noch nicht weiß, wie die vielfältigen Proteinbanden und ihre quantitativen Veränderungen hinreichend zu deuten und $\mathrm{zu}$ verstehen sind. Es werden noch weitere Untersuchungen vor allem auf dem Gebiet der klinischen Forschung mit dem Ziel einer weiteren Identifizierung der Serumproteine nötig sein, um die ungeahnten Möglichkeiten dieser Methode, welche Biochemiker den Medizinern in die Hände gelegt haben, bei der Diagnostik und der Therapiekontrolle voll ausnützen zu können.

Wir danken der Firma Wissenschaftlich-Technische Werkstätten $\mathrm{GmbH}$, Weilheim, die uns die Geräte für die analytische DiskElektrophorese freundlicherweise zur Verfügung gestellt hat. Frau Sibylle Suckart und Frl. UTE Krehn sind wir für ihre technische Mitarbeit sehr zu Dank verpflichtet.

\section{Literatur}

1. MAurer, H. R., Disk-Elektrophorese, 1. Aufl., Walter de Gruyter \& Co., Berlin (1968). - 2. Dietz, A. A. und T. Lubrano, Analytic. Biochem. 20, 246 (1967). - 3. 1. c. (1), S. 134. - 4. Lipoproteinemia Diagnosis, The A-P Test for Atherosclerosis Potential, Canalco Inc., Rockville. - 5. Wunderty, Ch. und H. H. MÄrkx, Dysproteinämien und Paraproteinämien, Schwabe \& Co., Basel-Stuttgart (1963). - 6. MAURER, H. R., Medizinal-Markt 16, 136 (1958). - 7. Journees Scientifiques de Tours: Appli- cations de l'electrophorese en gel de polyacrylamid, Tours (Frankreich) 21.-22. Nov. 1970, Annales de Biologic clinique (Paris) 29, 229 (1971). - 8. Grassmann, W. und K. Hannig, Hoppe-Seyler's Z. physiol. Chem. 290, 1 (1952). - 9. GrassaranN, W. und K. HaNNIG, Klin. Wschr. 32, 838 (1954). - 10. Strauch, L., Klin. Wschr. 34, 906 (1956). - 11. Wunderly, CH., Die Papierelcktrophoresc, 2. Aufl., H. R. Sauerländer \& Co., Aarau und Frankfurt/Main, S. 68-84 (1959). - 12. Lederer, E. und M. Lederer, Chromato- 
graphy, Elsevier Publ. Comp., 1. Aufl., Amsterdam, S. 91-93 (1955), 2. Aufl., S. 139-143 (1957). - 13. Brock, R. J., Paper Chromatography, Academic Press Inc., Publ., New York, S.33-50 (1952). - 14. Block, R. J., E. L. Durrum und G. Zweig, A Manual of Paper Chromatography and Paper Electrophoresis, 2. Aufl. Academic Press Inc., Publ. New York, S. 85-109 (1958). 15. Wunderly, CH., Paperelectrophoresis in M. Bier, Ed., Electrophoresis, Academic Press Inc. Publ., New York, S. 208-218 (1959). - 16. Schaub, M. C. und L. Strauch, Hoppe-Seyler's
Z. physiol. Chem. 349, 909 (1968). - 17. Felgenhauer, K., S. BACK und A. StaMmler, Klin. Wschr. 45, 371 (1967). 18. 1. c. (11), S. 81. - 19.1. c. (14), S. 95 . - 20.1. c. (14), S. 92. 21. 1. c. (11), S. 80. - 22. Joyce-Loebl, Method Sheet 112/A. 23. Isrcher, U. P., Med. Dissertation, Univ. München, 1966. Vgl. dazu l. c. (1) S. 128-129. - 24. Pastewka, J. V., A. T. Ness und A. C. Peacock, Clin. Chim. Acta, (Amsterdam) 14, 219 (1966). Vgl. ferner 1. c. (1), S. 131-133.

Dr.-Ing. L. Strauch c/o F. Hoffmann-La Roche \& Co. AG. Abt. VI/Med.

CH-4002 Basel (Schweiz) 